DOI 10. 18307/2017. 0105

(C) 2017 by Journal of Lake Sciences

\title{
浅水湖泊动力作用下水-土界面底泥起悬驱动力野外观测”
}

\author{
李一平 ${ }^{1,2}$, 王建威 ${ }^{* * *}$, 姜 龙 ${ }^{1}$, 唐春燕 ${ }^{4}$, 杜 薇 $^{1}$, 罗潋葱 ${ }^{3}$, 戴淑君 ${ }^{3}$ \\ ( 1 : 河海大学环境学院, 南京 210098) \\ (2: 河海大学浅水湖泊综合治理与资源开发教育部重点实验室, 南京 210098) \\ (3: 中国科学院南京地理与湖泊研究所,南京 210008) \\ (4: 新加坡南洋理工大学南洋环境与水源研究院环境模拟中心, 新加坡 637457)
}

\begin{abstract}
摘 要: 动力扰动引起的水-土界面沉积物悬浮是浅水湖泊蓝藻水华控制的难点, 本文基于声学高频流速仪、浊度仪、气 象、波浪等观测仪器获取的高时空分辨时间序列参量, 以太湖为例对动力扰动下的底泥起悬驱动力进行研究. 结果表明 风速小于 $3 \mathrm{~m} / \mathrm{s}$ 时, 水-土界面处平均悬浮物浓度为 $59 \mathrm{mg} / \mathrm{L}$, 波流综合切应力小于 $0.015 \mathrm{~N} / \mathrm{m}^{2}$, 底泥未起悬或在底床附 近极小范围内发生起悬; 风速在 $3 \sim 6 \mathrm{~m} / \mathrm{s}$ 时, 水-土界面处平均悬浮物浓度为 $103 \mathrm{mg} / \mathrm{L}$, 波浪产生的底切应力大部分情况 远大于湖流产生的切应力, 波流综合切应力处于 $0.015 \sim 0.25 \mathrm{~N} / \mathrm{m}^{2}$ 范围内, 底泥中等规模起悬; 风速大于 $6 \mathrm{~m} / \mathrm{s}$ 时, 水 - 土 界面处平均悬浮物浓度为 $174 \mathrm{mg} / \mathrm{L}$, 波浪产生的底切应力占据绝对的主导地位, 波流综合切应力大于 $0.25 \mathrm{~N} / \mathrm{m}^{2}$, 底泥大 规模起悬. 梅梁湾底泥起悬的临界切应力在 $0.015 \mathrm{~N} / \mathrm{m}^{2}$ 左右, 临界风速大约为 $3 \mathrm{~m} / \mathrm{s}$.
\end{abstract}

关键词: 水-土界面; 风; 底泥起悬;切应力;太湖;悬浮物浓度

\section{The driving force of sediment suspension on sediment-water interface in shallow lakes}

LI Yiping ${ }^{1,2}$, WANG Jianwei ${ }^{1 * *}$, JIANG Long ${ }^{1}$, TANG Chunyan $^{4}$, DU Wei ${ }^{1}$, LUO Liancong ${ }^{3}$ \& DAI Shujun $^{3}$ (1: College of Environment, Hohai University, Nanjing 210098, P.R.China)

(2: Key Laboratory of Integrated Regulation and Resource Development on Shallow Lakes, Ministry of Education, Hohai University, Nanjing 210098, P.R.China)

(3: Nanjing Institute of Geography and Liminology, Chinese Academy of Sciences, Nanjing 210008, P.R.China)

(4: Environmental Process Modelling Centre, Nanyang Environmental and Water Research Institute, Nanyang Technological University, 637457, Singapore)

\begin{abstract}
Sediment suspension on sediment-water interface caused by dynamic disturbance is the difficult issue in shallow lake eutrophication control. To study the driving force of sediment suspension in Meiliang Bay of Lake Taihu, a set of synchronous, highfrequency data of current, turbidity, wind, wave are obtained using the Acoustic Doppler Velocimeter, Optical Backscatter Sensor, PH-II Handheld weather stations, and RBRduo T.D. wave tide gauge. The results show that when the wind speed is less than $3 \mathrm{~m} / \mathrm{s}$, the average suspended solid concentration $(\mathrm{SSC}$ ) on sediment-water interface is approximately $59 \mathrm{mg} / \mathrm{L}$. The bottom shear stresses generated by waves is nearly equal to that generated by currents, where no sediment suspension can be found and the comprehensive shear stress generated by wind-induced waves and currents is less than $0.015 \mathrm{~N} / \mathrm{m}^{2}$. When the wind speed ranges from 3 to 6 $\mathrm{m} / \mathrm{s}$, the average SSC on sediment-water interface is approximately $103 \mathrm{mg} / \mathrm{L}$. The bottom shear stresses generated by waves is greater than that generated by currents in most cases, where a medium amount of sediment is suspended and the comprehensive shear stress generated by wind-induced waves and currents ranges from 0.015 to $0.25 \mathrm{~N} / \mathrm{m}^{2}$. When the wind speed exceeds $6 \mathrm{~m} / \mathrm{s}$ and the average SSC on sediment-water interface is approximately $174 \mathrm{mg} / \mathrm{L}$, the wave-generated shear stress plays an absolute leading role in large amount of sediment suspension and the comprehensive shear stress generated by wind-induced waves and cur-
\end{abstract}

* 国家自然科学基金项目 (51579071, 51379061)、中央高校基本科研业务项目基金项目(2014B07314)和江苏省高校 优势学科建设工程项目联合资助. 2016-03-20 收稿; 2016-05-11 收修改稿. 李一平 (1978 ), 男, 博士, 教授; E-mail : liyiping@hhu.edu.cn.

** 通信作者; E-mail: 1403860485@ qq.com. 
rents is larger than $0.25 \mathrm{~N} / \mathrm{m}^{2}$. The critical shear stress of sediment suspension in Meiliang Bay is approximately $0.015 \mathrm{~N} / \mathrm{m}^{2}$ while the critical wind speed is $3 \mathrm{~m} / \mathrm{s}$.

Keywords: Sediment-water interface; wind; bottom sediment suspension; shear stress; Lake Taihu; suspended solid concentration

大型浅水湖泊由于其水浅的特点, 易受到风动力的影响. 在风场的强迫作用下, 形成水面波动和底层湖 流的剪切作用 ${ }^{[1]}$, 导致水-土界面底泥再悬浮和水体污染的内源释放. 水-土界面是负责水体和沉积物之间 物质输送和交换的重要边界环境, 该界面处天然水体的物理、化学和生物特征具有显著的差异性,根据以往 经验来看底边界层内底泥起悬引起的内源释放是湖泊富营养化的主要来源 ${ }^{[2-3]}$, 这种动态释放严重影响了 湖泊的水质, 因此湖泊沉积物的起悬及内源释放日益受到重视. Lick ${ }^{[4]}$ 基于对美国 Great Lakes 开展的野外 观测, 发现悬浮物浓度 (Suspended Solid Concentration, SSC) 与底泥粒径大小及水一土界面切应力密切相关. 秦伯强等 ${ }^{[5-6]}$ 在野外调查观测与试验分析的基础上,研究了太湖沉积物悬浮的动力机制及相关的水动力过 程, 罗潋苟等 ${ }^{[1,7]}$ 借助波浪与水化学指标的实测资料也曾进行过类似的研究, 但前人设置的观测点往往远离 水-土界面, 使获取的水-土界面底泥临界驱动力的有效性大大降低. 逢勇等 ${ }^{[8]}$ 采用太湖湖区底泥, 根据波 浪水槽实验总结了各种扰动强度下太湖底泥的起动切应力,李一平等 ${ }^{[9]}$ 利用泥沙起动的理论模型计算了太 湖底泥在 3 种不同起动标准 (个别动、少量动、普遍动) 下的起动流速和起动切应力, 汤露露等 ${ }^{[10]}$ 利用 ECOMSED 模型对太湖湖流、波浪、沉积物的三维数值进行模拟. 总的来看, 前人的研究多侧重于室内试验和 数值模拟, 无论是室内试验还是数值模拟都有其不可避免的缺陷性. 室内试验难以保证底泥的原状性, 表层 上覆水体特征与野外真实情况存在差异, 且室内水槽实验基本上都只是构建了波浪或湖流单独作用的水动 力环境. 数值模拟由于假设条件及基于建模者的某些参数的取值, 往往造成与实际结果存在必然的差异. 在 过去有限的关于沉积物起悬的原位观测中, 受观测条件和观测仪器的限制, 很难得到水一土界面同一采样点 上的流速和 SSC 的高频时间序列, 使得沉积物再悬浮的驱动机制以及内源释放模式在国内外仍具有争 议 ${ }^{[11]}$. 近年来随着科技的发展, 基于声学设备 Acoustic Doppler Velocimeter (ADV)、Acoustic Doppler current profiler (ADCP) 及光学设备 Optical Backscatter Sensor(OBS) 探讨底边界层上悬浮物动力过程成为沉积物动 力学研究领域的热点, 目前这些仪器被广泛应用于海洋中, 在太湖中应用仍不多, Zheng 等 ${ }^{[12]}$ 借助 ADCP 研 究了太湖不同风速下的沉积物再悬浮过程,但其监测剖面的最底层距泥床仍有十几厘米的差距.

太湖是我国典型的大型浅水湖泊, 本研究以太湖为例, 选取典型湖区, 搭建野外观测平台, 配备能够观 测近底点流速和 SSC 高频时间序列的仪器, 以及气象、波浪等观测仪器. 基于获取的水体垂向及水-土界面 高时空分辨率的波浪、瞬时三维流速、SSC 等时间序列参量, 界定水一土界面底泥起悬的临界切应力与临界 风速,为今后湖泊内源污染治理措施的制定和悬浮泥沙扩散输移模型的建立提供理论支撑.

\section{1 仪器与方法}

\section{1 采样地点及时间}

太湖位于长江三角洲南缘, 是我国第三大淡水湖. 太湖水域面积 $2338 \mathrm{~km}^{2}$, 南北长 $68.5 \mathrm{~km}$, 东西宽 34 $\mathrm{km}$, 多年平均水深 $1.89 \mathrm{~m}$. 太湖的风速可从 $0 \mathrm{~m} / \mathrm{s}$ 变化至 $10 \mathrm{~m} / \mathrm{s}$, 春、夏季平均风速为 $4.3 \mathrm{~m} / \mathrm{s}$, 秋、冬季平均 风速为 $0.9 \mathrm{~m} / \mathrm{s}^{[13]}$. 环湖河流共有 228 条, 2014 年人湖水量为 $101.56 \times 10^{8} \mathrm{~m}^{3}$ 、出湖水量为 $104.06 \times 10^{8} \mathrm{~m}^{3}$. 湖 西区仍是人湖水量的主要来源, 全年有 $71 \%$ 的人湖水量来自湖西区. 梅梁湾系太湖北部一半封闭湖湾, 南北 长约 $14 \mathrm{~km}$, 东西宽约 $7 \mathrm{~km}$, 水面面积约 $129 \mathrm{~km}^{2}$, 平均水深 $2.1 \mathrm{~m}$, 是无锡市主要旅游区及水源地. 梅梁湾主 要人湖河道有 3 条, 为武进港、直湖港和梁溪河.

本研究于 2014 年 4 月 $23-27$ 日在太湖梅梁湾与湖心区交界处拖山附近 $\left(31^{\circ} 23^{\prime} 34.29^{\prime \prime} \mathrm{N}, 120^{\circ} 9^{\prime} 24.86^{\prime \prime} \mathrm{E}\right)$ (图 1) 搭建的野外观测平台进行定点连续观测, 测点北部为梅梁湾湖湾区, 南部为开阔湖区. 观测期间太湖 处于高水位,水位在 $2.63 \sim 2.81 \mathrm{~m}$ 范围内变化,平均水深 $2.7 \mathrm{~m}$.

\section{2 观测方案及仪器配置}

本研究现场同步高频监测了风场、湖流、波浪、浊度等数据. 风速和风向测定采用固定于风浪观测支架 
上的 PH- II Handheld 手持式气象站和 PHWD 风向传感 器, 频率为 $10 \mathrm{~min}$, 距离水面 $5 \mathrm{~m}$. 流速采用单点声学高 频流速仪 ADV Ocean (工作频率为 $5 \mathrm{MHz}$ ) 进行连续观 测. 其探头靠近湖底, 实际测点距离底部 $5 \mathrm{~cm}$, 监测频 率 $10 \mathrm{~Hz}$, 获取底层距离泥床 $5 \mathrm{~cm}$ 范围内的三维流速序 列. 波浪采用 MIDAS DWR 方向波潮仪连续监测, 采样 间隔为 $5 \mathrm{~min}$, 仪器固定在水下 $95 \mathrm{~cm}$, 采用线性波理论 分析方法, 64 位数字处理技术, 实时测量有效波高、波 周期、平均波向及其他统计要素. 浊度采用 2 台 OBS-3A 型浊度仪自动采集高频浊度数据, 监测频率均为 $3 \mathrm{~min}$ 一次. 上层 $\mathrm{OBS}($ 测量范围为 $0 \sim 250 \mathrm{NTU}$ ) 放置在水面 以下 $95 \mathrm{~cm}$, 用于监测近表层浊度值; 底层 $\mathrm{OBS}$ (测量范 围为 $0 \sim 1000 \mathrm{NTU}$ ) 放置在与 ADV Ocean 同高度处, 用 于监测水-土界面浊度值, 此外通过真空原现场抽滤采 集近表层与底层的水样,同时记录下不同深度水样的采 集时间,采样频率约为 $3 \mathrm{~h}$ 一次,一天进行 4 次,水样在 实验室进行分析得到 SSC.

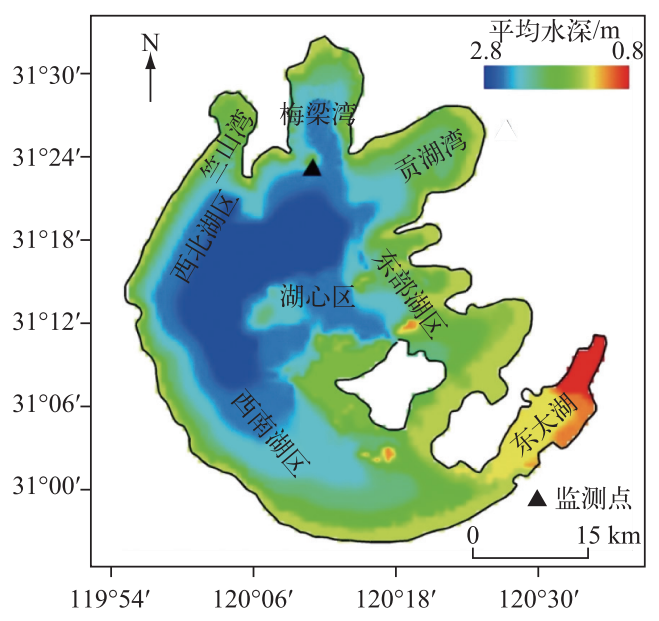

图 1 太湖梅梁湾监测点位

Fig.1 Location of the study site at Meiliang Bay in Lake Taihu

\section{3 数据处理}

1.3.1 湖泊水一土界面由波浪产生的切应力 水表面质点沿椭圆轨道运动, 当波进人浅水区时, 水质点的椭 圆形路径逐渐压扁呈水平直线, 尤其是在没有垂直流的底部. 随着轨道被压扁, 水质点的运动逐渐变为水平 震荡. 底床附近最大的波动轨道速度可用下式表示:

$$
\begin{gathered}
u_{\mathrm{w}}=\frac{\pi \cdot H_{\mathrm{s}}}{T_{\mathrm{s}} \cdot \sinh \left(\frac{2 \pi \cdot h}{L_{\mathrm{s}}}\right)} \\
L_{\mathrm{s}}=\left(\frac{g \cdot T_{\mathrm{s}}{ }^{2}}{2 \pi}\right) \tanh \left(\frac{2 \pi \cdot h}{L_{\mathrm{s}}}\right)
\end{gathered}
$$

式中, $u_{\mathrm{w}}$ 为底床附近最大的波动轨道速度 $(\mathrm{m} / \mathrm{s}) ; h$ 代表观测点水深 $(\mathrm{m}) ; H_{\mathrm{s}}$ 为有效波的波高 $(\mathrm{m}) ; T_{\mathrm{s}}$ 为有效 波的周期 $(\mathrm{s}) ; L_{\mathrm{s}}$ 为有效波的波长, 其初值可取与研究区域中有效波长相近的数值, 经多次迭代达到稳定可 输人式 (1) 参与计算.

基于 Grand \& Madsen 系统地提出的水底边界层切应力计算的基本理论,波切应力 $\tau_{w}$ 可采用下式进行 计算 ${ }^{[14]}$ :

$$
\tau_{\mathrm{w}}=0.5 \rho \cdot f_{\mathrm{w}} \cdot u_{\mathrm{w}}^{2}
$$

式中, $\tau_{\mathrm{w}}$ 表示波切应力 $\left(\mathrm{N} / \mathrm{m}^{2}\right) ; \rho$ 为水体密度 $\left(\mathrm{kg} / \mathrm{m}^{3}\right) ; u_{\mathrm{w}}$ 为底床附近最大的波动轨道速度 $(\mathrm{m} / \mathrm{s}) ; f_{\mathrm{w}}$ 表示波 摩擦系数, 无量纲, 其与水底粗䊅度和雷诺数有关. $f_{\mathrm{w}}$ 计算公式为 ${ }^{[15]}$ :

$$
\begin{gathered}
f_{\mathrm{w}}=\exp \left[5.2\left(A_{\delta} / K_{\mathrm{s}}\right)^{-0.19}-6.0\right] \\
f_{\mathrm{w}, \max }=0.3 \quad\left(\text { 若 } A_{\delta} / K_{\mathrm{s}} \leqslant 1.57\right)
\end{gathered}
$$

式中, $K_{\mathrm{s}}$ 为湖底物理粗粘度, 难以通过野外观测获得其真实值, 本文参照 Luetich 等 ${ }^{[16]}$ 、Hawley ${ }^{[17]}$ 和秦伯强 等 ${ }^{[6]}$ 的取法, 取 $0.0002 \mathrm{~m} ; A_{\delta}$ 为近底波浪质点振幅 $(\mathrm{m})$, 通过线性波理论确定:

$$
A_{\delta}=\frac{H_{\mathrm{s}}}{2 \sinh \left(\frac{2 \pi}{L_{\mathrm{s}}} h\right)}
$$

1.3.2 湖泊水一土界面由湖流产生的切应力 湖流产生的切应力主要是根据流速的对数分布, 与摩阻流速的 平方呈正比, 其计算方法为 ${ }^{[17-18]}$ :

$$
\tau_{c}=\rho\left(u_{\mathrm{b}}^{*}\right)^{2}
$$




$$
u_{\mathrm{b}}^{*}=\frac{k \cdot u_{z}}{\ln \frac{z}{K_{\mathrm{s}}}}
$$

式中, $\tau_{\mathrm{c}}$ 表示流切应力 $\left(\mathrm{N} / \mathrm{m}^{2}\right) ; u_{\mathrm{b}}^{*}$ 为流摩阻流速 $(\mathrm{m} / \mathrm{s}) ; k$ 为卡曼常数, 取 $0.4 ; u_{z}$ 表示湖底以上 $z$ 高处 $(\mathrm{m})$ 的 湖流速度 $(\mathrm{m} / \mathrm{s})$.

1.3.3 湖泊水一土界面由波流产生的综合切应力 波流相互作用生成了强度和方向都发生变化的切应力. 综 合切应力 $\tau_{\mathrm{cw}}$ 计算方法为 $^{[19]}$ :

$$
\tau_{\mathrm{cw}}=\tau_{\mathrm{w}} \sqrt{1+\left(\tau_{\mathrm{c}} / \tau_{\mathrm{w}}\right)^{2}+2\left(\tau_{\mathrm{c}} / \tau_{\mathrm{w}}\right)|\cos (\delta-\alpha)|}
$$

式中, $\tau_{\mathrm{cw}}$ 表示波流产生的综合切应力 $\left(\mathrm{N} / \mathrm{m}^{2}\right) ; \alpha$ 和 $\delta$ 是波和流的方向.

1.3.4 湖泊水一土界面浊度的标定 通过实验室分析得到 SSC, 建立起其与水一土界面浊度的关系, 将浊度时 间序列转换成 SSC 时间序列. OBS 测得的浊度在标定范围内 $(0 \sim 1000 \mathrm{NTU})$ 存在良好的线性关系, 相关系数 $R^{2}$ 为 0.9375 (图 2). 秦伯强等在野外调查观测与实验室试验分析的基础上, 发现了太湖水-土界面物质交换 主要发生在沉积物表层 5 10 cm 范围内 ${ }^{[6]}$. 罗潋葱等对太湖不同湖区沉积物粒径分析结果表明深度达到 $10 \mathrm{~cm}$ 的沉积物粒径比较稳定, 而深度在 $10 \mathrm{~cm}$ 以下的会出现波动 ${ }^{[20]}$. 因此可初步判定本研究中野外观测 点位悬浮物粒径组成相对比较稳定, OBS 观测得到的浊度与县浮物浓度 SSC 的线性关系比较可靠, 可通过 浊度推演水体悬浮物浓度.

\section{2 结果与讨论}

\section{1 动力条件下水体 SSC 垂向变化特征}

采用监测时间段每隔 $1 \mathrm{~h}$ 的同步数据,进行风速与上层和底层 SSC 的关系分析 (图 3 ). 结果表明,在风 速小于 $3 \mathrm{~m} / \mathrm{s}$ 时, 上层和底层 $\mathrm{SSC}$ 变化范围相差不大, 集中在 $50 \sim 90 \mathrm{mg} / \mathrm{L}$ 左右, 这是由于上层悬浮物中有 机物占一半以上, 因此在风速很小时, 悬浮物同样可以浮到上层 ${ }^{[5]}$. 风速在 $3 \sim 6 \mathrm{~m} / \mathrm{s}$ 时, SSC 波动范围较大, 表层集中在 $54 \sim 140 \mathrm{mg} / \mathrm{L}$, 最大增加到小风速的 $2 \sim 3$ 倍, 底层集中在 $45 \sim 238 \mathrm{mg} / \mathrm{L}$, 最大增加到小风速的 $4 \sim 5$ 倍, 底层 SSC 变化范围明显大于表层, 这种情况可能是因为底泥发生起县. 在风速大于 $6 \mathrm{~m} / \mathrm{s}$ 后, 整个 水体的 SSC 大幅增加, 表层集中在 30 210 mg/L, 底层集中在 45 296 mg/L, 底层水体 SSC 变化要比表层水 体剧烈得多, 平均 SSC 底层比表层大 $7 \mathrm{mg} / \mathrm{L}$ 左右. 风浪扰动强度, 除了与风速相关外, 还与风的吹程有关, 而风的吹程与风向密切相关, 因此有必要对观测期间不同风向下 (E、ENE、ESE、SE、SSE、W、WNW) 底层 SSC

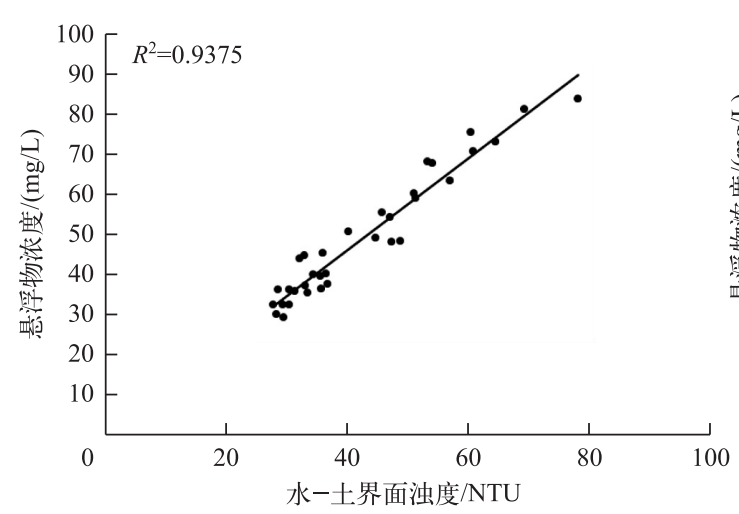

图 2 现场水样抽滤物质浓度标定 水一土界面浊度

Fig. 2 Calibration of OBS turbidity in the bottom layer with bottle samples

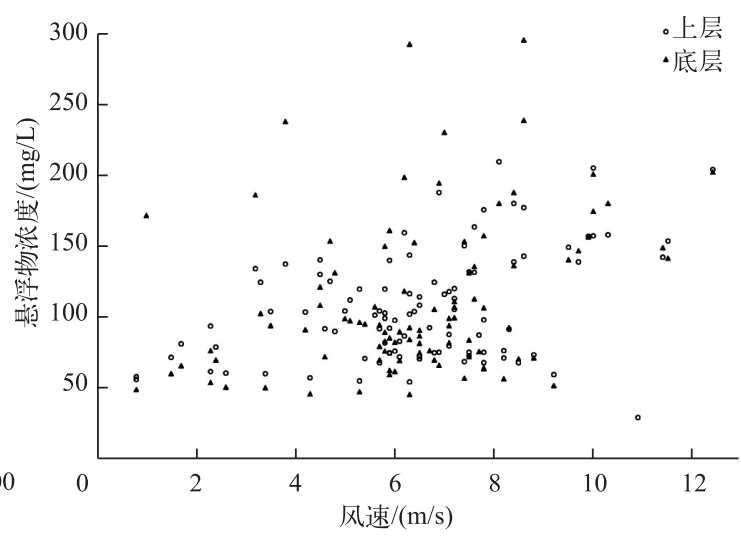

图 3 上层和底层悬浮物浓度与 同时刻风速的变化关系

Fig. 3 The relationship between SSC in the surface and bottom layers and wind speed 
与风速的变化关系进行分析 (图 4). 在 W 和 WNW 风场中, 即使风速大于 $6 \mathrm{~m} / \mathrm{s}, \mathrm{SSC}$ 变化也并不明显, 这主
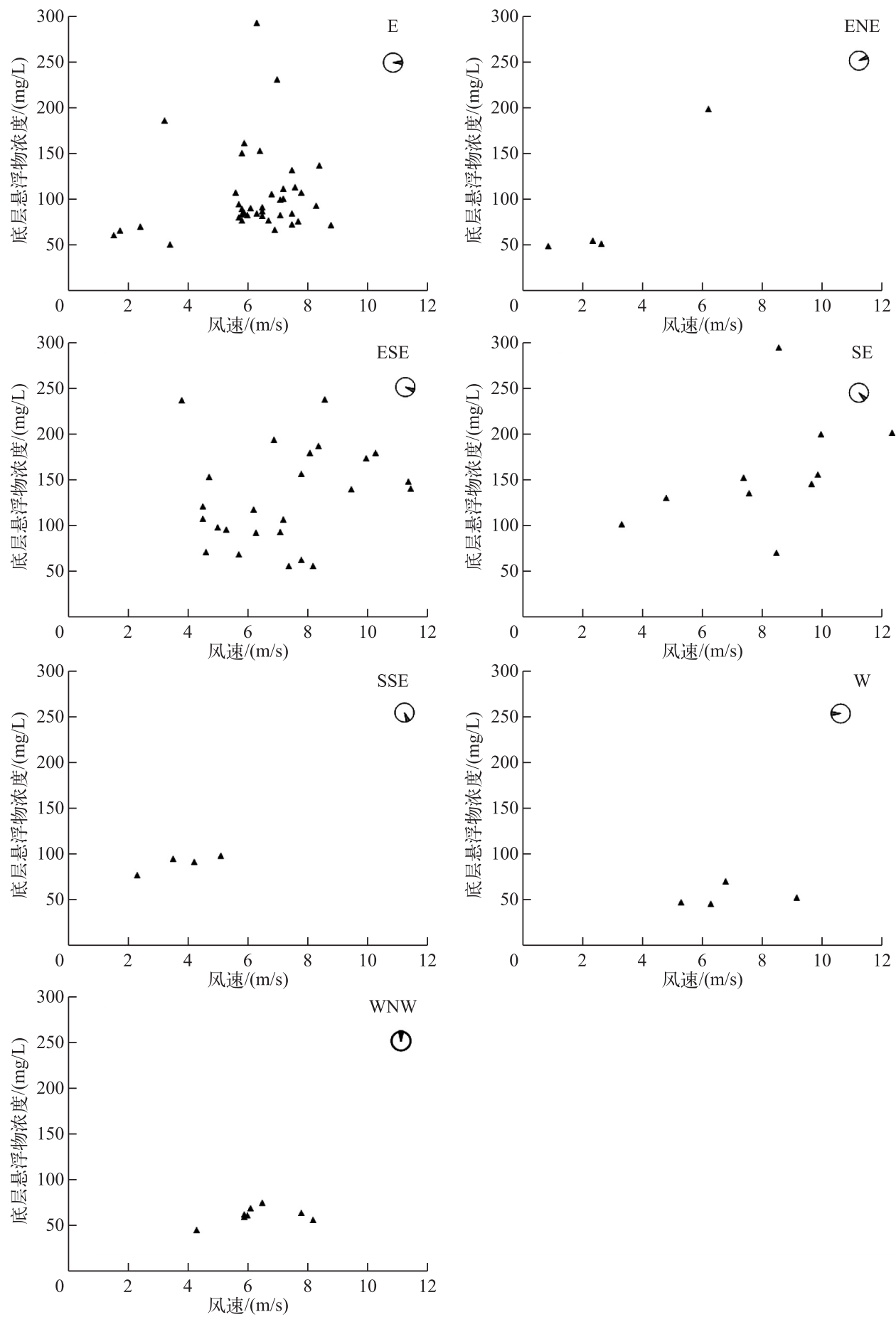

图 4 观测期间不同风向情况下风速与底层悬浮物浓度的关系

Fig.4 The relationship between SSC in the bottom layer and wind speed with different wind directions during the observation 
要是因为 $\mathrm{W}$ 和 $\mathrm{WNW}$ 风场的吹程短, 风区长度小, 即使大风速下气一水界面处也没有产生很大的能量, 在观 测到的其他风向下,SSC 的整体变化趋势随风速的增大而增大. E、ESE、SE 是观测期间出现频率较高且风区 长度相对较大的风场, 综合 3 种风向下底层 SSC 和风速的变化关系来看, 也是近似符合风速 $3 \sim 6 \mathrm{~m} / \mathrm{s}$ 时 SSC 初步增加、风速大于 $6 \mathrm{~m} / \mathrm{s}$ 时 SSC 大幅增加的规律的. 在 E 和 ESE 风场中, 相对仍有个别代表小风速大 SSC 和大风速小 SSC 的点, 这可能是由于水面波动对风场变化的滞后效应, 也有可能风速的瞬时变化率才是影 响沉积物起悬的关键因素.

\section{2 风驱动下太湖水一土界面综合切应力特征及波流贡献率的界定}

波切应力、流切应力和综合切应力随风速的变化而变化, 且三者变化趋势与风速的变化趋势基本一致, 只 是在大风速的情况下, 产生较大的风浪, 波浪产生的切应力和综合切应力在量级上远大于湖流产生的切应力 (图 5). 只有在风速较小的情况下 (例如,4 月 26 日 6:00-8:00), 湖流产生的切应力与波浪产生的切应力、综 合切应力相对接近 (切应力在 $0.005 \sim 0.02 \mathrm{~N} / \mathrm{m}^{2}$ 左右). 监测期间综合切应力的大小及变化趋势与波浪切应力 基本一致,这主要是由于大部分时间内波浪产生的切应力占主导作用, 具体可以通过比较波切应力与流切应 力比值来反映波、流切应力对综合切应力的贡献 (图 6). 当风速小于 $3 \mathrm{~m} / \mathrm{s}$ 时, 波切应力与流切应力的比值 (以 下简称切应力的比值) 小于 1 和大于 1 的情况都有出现, 说明小风速情况下流切应力对水-土界面沉积物悬浮 的贡献是可能大于波切应力的. 当风速在 $3 \sim 6 \mathrm{~m} / \mathrm{s}$ 之间时, 切应力的比值大多数时候大于 1 , 最大达到 17.7 , 即 大部分情况下水-土界面沉积物悬浮是由波切应力引起的. 当风速大于 $6 \mathrm{~m} / \mathrm{s}$ 时, 切应力的比值始终大于 1 , 波 浪产生的切应力在底泥起悬的动力过程中占据了绝对主导地位, 此时可以忽略流切应力.
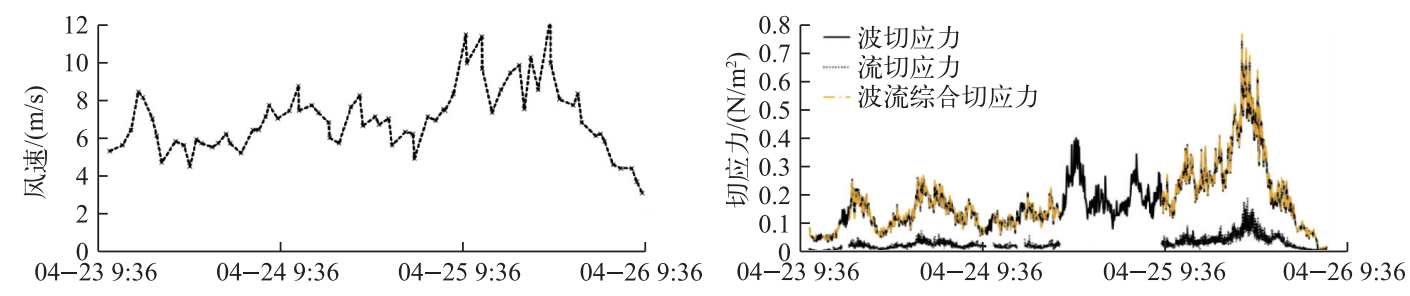

图 52014 年观测期间风速与底层波切应力、流切应力、综合切应力的比较

Fig. 5 Comparison of wind speed and shear stress induced by wave, current and combination during the observation of 2014

\section{3 动力条件下太湖水一土界面底泥起悬临界切应力与临界风速的确定}

上文中切应力与风速的变化关系同风速与底层 SSC 的变化关系在量上保持高度一致,即当风速达到 $3 \sim 6 \mathrm{~m} / \mathrm{s}$ 以上时, 剪切力迅速增强, 底层 SSC 也随即增大, 这可能由于底泥颗粒物的内聚力被打破. 为了进 一步说明风引起的底部剪切力与底层 SSC 的关系, 将两者进行曲线拟合 (图 7). 本次观测中, 按照悬浮物浓 度随底层剪切力的整体变化规律, 将底泥起悬分为 3 个阶段:

A 阶段: 底泥未起悬或在底床附近极小范围内发生起悬, $\tau_{\mathrm{cw}}<0.015 \mathrm{~N} / \mathrm{m}^{2}$. 此时床面处于稳定状态, 水土界面处 SSC 变化不大,平均 $\mathrm{SSC}$ 为 $59 \mathrm{mg} / \mathrm{L}$.

B 阶段: 底泥中等规模起悬, $0.015 \leqslant \tau_{\mathrm{cw}}<0.25 \mathrm{~N} / \mathrm{m}^{2}$. 此时底床受到破坏, 水一土界面处 SSC 开始增大, 随 着剪切力的增加, 不断掀起底泥, 悬浮物浓度增速加快, 平均 SSC 达到 $103 \mathrm{mg} / \mathrm{L}$.

C 阶段: 底泥大规模起悬, $\tau_{\mathrm{cw}} \geqslant 0.25 \mathrm{~N} / \mathrm{m}^{2}$. 此时床面受到大程度破坏, 水-土界面水体很快便完全浑浊, 平均 $\mathrm{SSC}$ 达到 $174 \mathrm{mg} / \mathrm{L}$.

以底泥中等规模起悬为标准, 本次观测得到的临界剪切力在 $0.015 \mathrm{~N} / \mathrm{m}^{2}$ 左右, 其与水深、流速、底泥特 性等有关. 沉积物悬浮的临界剪切力一般取值范围为 $0.01 \sim 0.1 \mathrm{~N} / \mathrm{m}^{2[18,21-22]}$, 本次观测得到的临界切应力在 此范围之内. 将不同风速下综合切应力与水一土界面沉积物悬浮临界切应力相比较可知, 当风速大于 $3 \mathrm{~m} / \mathrm{s}$ 时, 切应力随风速变化呈线性上升趋势, 综合切应力的数值远大于临界切应力, 该过程对应底泥中等规模与 大规模起悬. 当风速小于 $3 \mathrm{~m} / \mathrm{s}$ 时, 综合切应力与风速变化的相关性较弱, 综合切应力的数值基本处于临界 切应力以下, 该过程底泥处于未起悬或者在底床附近极小范围内起悬的状态 (图 8). 因此本次观测得到的 
泥沙起悬临界风速大约为 $3 \mathrm{~m} / \mathrm{s}$.

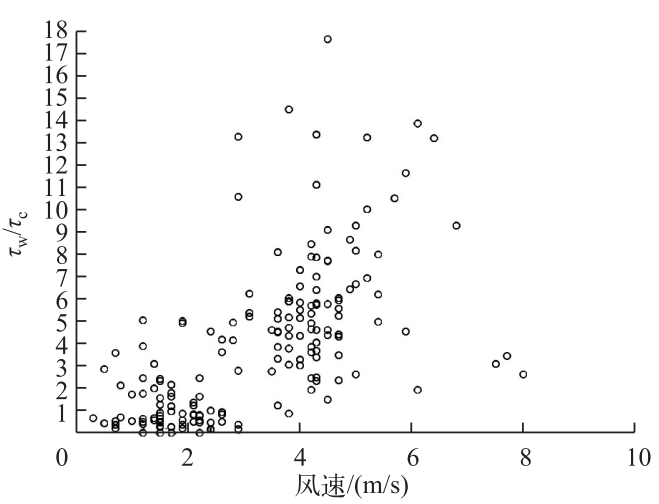

图 6 观测期间波切应力与 流切应力的比值随风速的变化关系

Fig. 6 The relationship between the wind and ratio of wave-induced shear stress/currentinduced shear stress during the observation

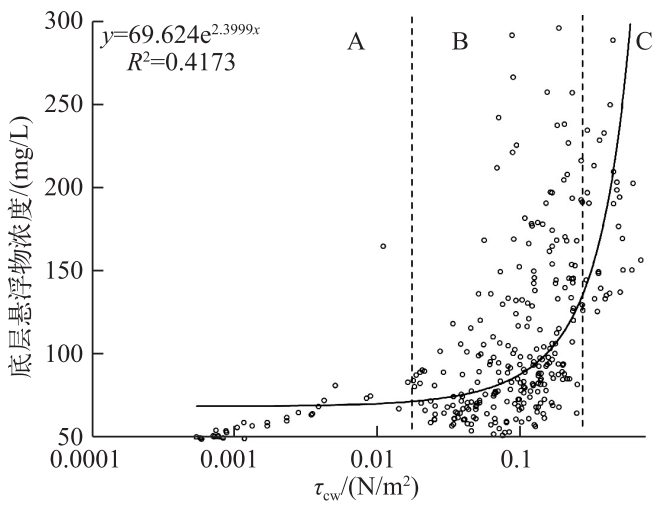

图 7 观测期间底层 $\mathrm{SSC}$ 与波流 综合切应力之间的关系

Fig.7 The relationship between SSC in the bottom layer and shear stress generated by wind-induced waves and currents during the observation
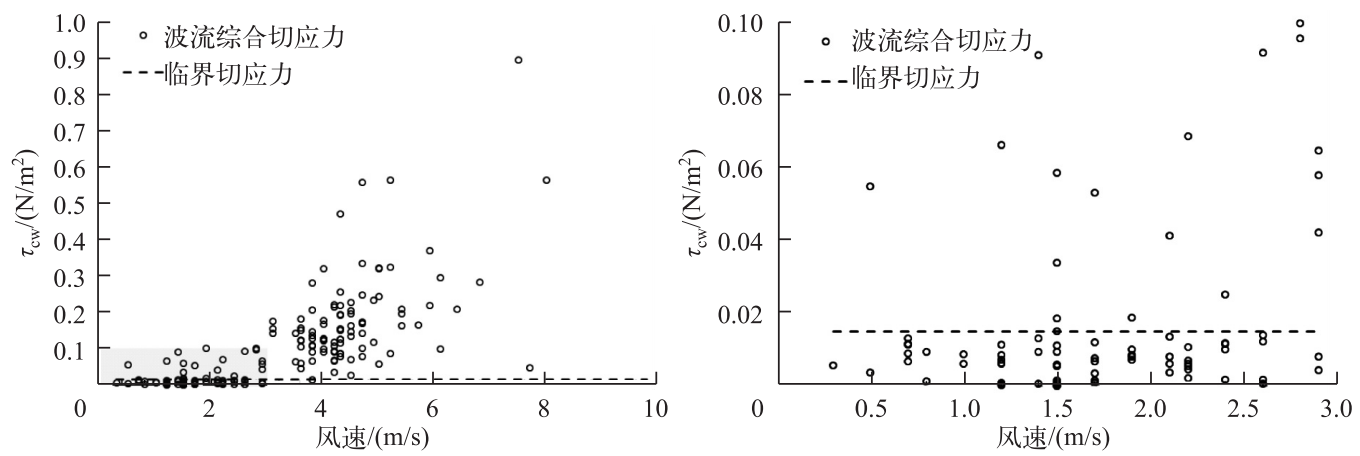

图 8 不同风速下波流综合切应力与底层沉积物悬浮临界切应力的比较

Fig. 8 Comparison of shear stress generated by wind-induced waves and currents and the critical stress of sediments on sediment-water interface

\section{3 讨论}

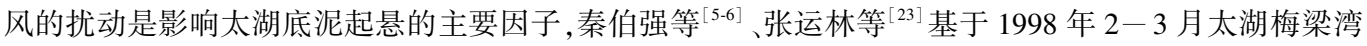
SSC 的观测发现深层水悬浮物浓度要大于表层水, 随着风速的增强, 垂直方向的差异更加明显, 这与本文的 结果是一致的. 以上结果同时证明由于风浪对沉积物的扰动引起的再悬浮在水一土界面尤为强烈, 因此在风 浪条件下是无法用表层 SSC 计算整个水柱的悬浮物总量的,前人通过高精度分层同步采样器曾获得类似的 结果 ${ }^{[24]}$. 风并不是直接作用于底泥, 而是先作用于湖面, 使得水动力条件发生变化, 然后水-土界面的沉积 物在波浪和湖流综合切应力作用下产生悬浮、输移. 罗潋葱 ${ }^{[7]}$ 、秦伯强 ${ }^{[6]}$ 等认为小风速下波浪与湖流产生的 切应力大致相当, 但是当风速较大时, 波浪作用占主导, 本文的结果与其相似. 在获得了底层切应力及 SSC 后, 可通过分析 SSC 的变化来获得临界切应力. 秦伯强等 ${ }^{[6]}$ 在实验室获得的底泥的临界起动切应力约为 $0.037 \mathrm{~N} / \mathrm{m}^{2}$, 临界风速为 $4 \mathrm{~m} / \mathrm{s}$, 当风速达到 $6.5 \mathrm{~m} / \mathrm{s}$ 时, 沉积物发生大规模悬浮. 前人获得的临界切应力比 本文的结果大, 主要是因为秦伯强等将沉积物起动标准定义为普遍动, 在进行室内水槽实验时无法保证底 
泥的原状性且只考虑了波浪的单一作用. Zheng 等 ${ }^{[12]}$ 认为底泥起悬的临界风速为 $3.7 \sim 4.0 \mathrm{~m} / \mathrm{s}$, 临界切应力 为 $0.011 \mathrm{~N} / \mathrm{m}^{2}$, 底泥大规模起悬时风速达到 $6 \mathrm{~m} / \mathrm{s}$, 本文结果与之相近, 但临界风速比其结果略大, 可能是因 为 Zheng 等测量得到的 SSC 数列较少, 与风速拟合的精确度有限所致. 罗潋苟等 ${ }^{[1,7]}$ 在分析波浪与湖流对沉 积物再悬浮的不同影响时指出当风速达 $5 \mathrm{~m} / \mathrm{s}$ 以上时, SSC 将显著增加. 罗潋苟得到的起悬风速之所以明显 大于本文结果是因为其参考标准是剖面 SSC 变化, 且因观测手段和资料有限, 仅仅获取了湖底以上 $0.5 \mathrm{~m}$ 处 的流速, 并不能真正反映水一土界面处流速及相应切应力的变化情况, 而本文不但观测到了水一土界面以上 5 $\mathrm{cm}$ 的三维高频流速序列, 而且获取了相应高度的高频 SSC 序列, 因此本次监测获得的临界切应力、临界风 速作为底泥起悬的判别标准更具有合理性和代表性, 可为今后悬浮泥沙扩散输移的数值模拟提供更加准确 的参数依据. 从微观紊动角度来看, 三维高频流速序列的垂向流速对沉积物再悬浮也是有影响的, 床面上的 紊动作用使得低流速水团可以直接挟带一部分泥沙颗粒上升到主流区, 但本文的侧重点为与底泥悬浮相关 的平均态动力参数, 因此与底泥悬浮相关的脉动参数可以在以后的工作中进行更为细致的研究. 窦国仁 ${ }^{[25]}$ 认为泥沙起动可分为 3 种状态, 即个别动、少量动和普遍动, 并将少量动定为泥沙起动的标准, 本文要说明 的是荬先生研究的主要是泥沙颗粒从推移运动到悬移运动的状态变化过程, 但是太湖泥沙不经跃移过程, 直接从静止状态转为悬移运动是存在的, 这时候得到的切应力实为临界扬动切应力, 这种过程可能和床面 附近的紊动作用有关联.

\section{4 结论}

基于野外原位观测实验获取的风场、流场、波浪场、浊度等数据发现在风速小于 $3 \mathrm{~m} / \mathrm{s}$ 时, 上层和水一土界 面处 SSC 变化集中在 50 90 mg/L 左右, 波、流切应力相当, $\tau_{\mathrm{cw}}<0.015 \mathrm{~N} / \mathrm{m}^{2}$, 底泥未起悬或者在底床附近极小 范围内发生起悬; 风速在 3 6 m/s 时, 上层 SSC 集中在 54 140 mg/L, 水-土界面处 SSC 集中在 $45 \sim 238 \mathrm{mg} / \mathrm{L}$, 波浪产生的切应力大部分情况远大于湖流产生的切应力, $0.015 \leqslant \tau_{\mathrm{cw}}<0.25 \mathrm{~N} / \mathrm{m}^{2}$, 底泥中等规模起悬; 风速大于 $6 \mathrm{~m} / \mathrm{s}$ 时, 上层 SSC 集中在 30 210 mg/L, 水-土界面处 SSC 集中在 $45 \sim 296 \mathrm{mg} / \mathrm{L}$, 水-土界面处水体 SSC 变化 要比上层水体剧烈得多, 波浪产生的切应力占据绝对的主导地位, $\tau_{\mathrm{cw}} \geqslant 0.25 \mathrm{~N} / \mathrm{m}^{2}$, 底泥大规模起悬. 梅梁湾底 泥起悬的临界切应力在 $0.015 \mathrm{~N} / \mathrm{m}^{2}$ 左右, 临界风速大约为 $3 \mathrm{~m} / \mathrm{s}$. 本研究反映出浅水湖泊水一土界面的底泥易 受到扰动而发生起悬, 在本文的基础上研究太湖及其他大型浅水湖泊沉积物再悬浮驱动机理时必须更多地考 虑具体观测位置的地形、地貌等因素,不同的地形、地貌有着不同的底泥特征及风浪孕育程度.

\section{5 参考文献}

[ 1 ] Luo Liancong, Qin Boqiang. Comparison between wave effects and current effects on sediment resuspension in Lake Taihu. Hydrology, 2003, 23(3) : 1-4. [罗潋葱, 秦伯强. 太湖波浪与湖流对沉积物再悬浮不同影响的研究. 水文, 2003, $\mathbf{2 3}(3): 1-4$. ]

[ 2 ] Bailey MC, Hamilton DP. Wind induced sediment resuspension: A lake-wide model. Ecological Modelling, 1997, 99(2/ $3)$ : 217-228. DOI: 10.1016/S0304-3800(97)01955-8.

[ 3 ] Zhu G, Qin B, Gao G. Direct evidence of phosphorus outbreak release from sediment to overlying water in a large shallow lake caused by strong wind wave disturbance. Science Bulletin, 2005, 50(6) : 577-582. DOI:10.1007/BF02897483.

[ 4 ] Lick W. The flocculation, deposition, and resuspension of fine-grained sediments. In: Depinto JV, Lick W, Paul JF eds. Transport and Transformation of Contaminants Near the Sediment-water Interface. New York: Springer, 1994: 35-37.

[ 5 ] Qin Boqiang, Hu Weiping, Chen Weimin et al. Studies on the hydrodynamic processes and related factors in Meiliang Bay, Northern Taihu Lake, China. J Lake Sci, 2000, 12(4) : 327-334. DOI: 10.18307/2000.0406. [秦伯强, 胡维平, 陈伟民等. 太湖梅梁湾水动力及相关过程的研究. 湖泊科学, 2000, 12(4) : 327-334.]

[6] Qin Boqiang, Hu Weiping, Gao Guang et al. Dynamics of sediment resuspension and the conceptual schema of nutrient release in the large shallow Lake Taihu, China. Science Bulletin, 2003, (17) : 1822-1831. [秦伯强, 胡维平, 高光等. 太 湖沉积物悬浮的动力机制及内源释放的概念性模式. 科学通报, 2003, (17) : 1822-1831.]

[ 7 ] Luo Liancong, Qin Boqiang, Hu Weiping et al. Sediment re-suspension under different hydrodynamic disturbances in Lake Taihu. J Lake Sci, 2004, 16(3) : 273-276. DOI: 10.18307/2004.0313. [罗欲葱, 秦伯强,胡维平等. 不同水动力扰动 
下太湖沉积物的悬浮特征. 湖泊科学, 2004, 16(3) : 273-276.]

[ 8 ] Pang Yong, Zhuang Wei, Han Tao et al. Experiment and model simulation of suspended solids in Taihu Lake under windwave disturbance. Environmental Science, 2008, 29(10): 2743-2748. [ 逢勇, 庄巍, 韩涛等. 风浪扰动下的太湖悬浮物 实验与模拟. 环境科学, 2008, 29(10): 2743-2748.]

[ 9 ] Li Yiping, Pang Yong, Luo Liancong. Experimental and numerical study on the transfer process of suspended matter in the interaction of wave and current in Taihu Lake. Advances in Water Science, 2009, 20(5): 701-706. [李一平, 逢勇, 罗潋 葱. 波流作用下太湖水体悬浮物输运实验及模拟. 水科学进展, 2009, 20(5): 701-706. ]

[10] Tang Lulu, Wang Peng, Yao Qi. Three-dimensional numerical simulation of current, waves and sediment transport in Taihu Lake. Water Resources Protection, 2011, 27(2) : 1-5. [汤露露, 王鹏,姚琪. 太湖湖流、波浪、沉积物的三维数值 模拟. 水资源保护, 2011, 27(2): 1-5. ]

[11] Qin Boqiang. Progress and prospect on the eco-environmental research of Lake Taihu. J Lake Sci, 2009, 21 (4) : 445-455. DOI : 10.18307/2009.0401. [ 秦伯强. 太湖生态与环境若干问题的研究进展及其展望. 湖泊科学, 2009, 21(4): 445-455.]

[12] Zheng S, Wang P, Wang C et al. Sediment resuspension under action of wind in Taihu Lake, China. International Journal of Sediment Research, 2015, 30(1) : 48-62. DOI : 10.1016/S1001-6279(15)60005-1.

[13] Qian Haozhong. The influence of wind field to the spatial distribution of chlorophyll-a concentration [Dissertation]. Nanjing: Nanjing University of Information Science \& Technology, 2012. [ 钱吴钟. 风场对太湖叶绿素 a 空间分布的影响规 律研究 [学位论文]. 南京:南京信息工程大学, 2012.]

[14] Grant WD, Madsen OS. Combined wave and current interaction with a rough bottom. Journal of Geophysical Research: Oceans $(1978$ - 2012), 1979, 84(C4) : 1797-1808. DOI: 10.1029/JC084iC04p01797.

[15] Jiang W, Pohlmann T, Sündermann J et al. A modelling study of SPM transport in the Bohai Sea. Journal of Marine Systems, 2000, 24(3) : 175-200. DOI: 10.1016/S0924-7963(99)00071-8.

[16] Luettich RA, Harleman DR, Somlyody L. Dynamic behavior of suspended sediment concentrations in a shallow lake perturbed by episodic wind events. Limnology and Oceanography, 1990, 35 (5) : 1050-1067. DOI: 10.4319/lo. 1990.35. 5.1050 .

[17] Hawley N. Sediment resuspension near the Keweenaw Peninsula, Lake Superior during the fall and winter $1990-1991$. Journal of Great Lakes Research, 2000, 26(4) : 495-505. DOI: 10.1016/S0380-1330( 00) 70711-8.

[18] Sheng YP, Lick W. The transport and resuspension of sediments in a shallow lake. Journal of Geophysical Research, 1979, 84(C4) : 1809-1826. DOI: 10.1029/JC084iC04p01809.

[19] Rosales P, Ocampo-Torres FJ, Osuna P et al. Wave-current interaction in coastal waters: Effects on the bottom-shear stress. Journal of Marine Systems, 2008, 71(1) : 131-148. DOI : 10.1016/j.jmarsys.2007.06.006.

[20] Luo Liancong, Zhang Fabing. Application of ADP in the study of sediment resuspension in Lake Taihu. J Lake Sci, 2003, 15(4) : 331-338. DOI: 10.18307/2003.0406. [ 罗潋葱, 张发兵. ADP 在太湖沉积物再悬浮分析中的应用. 湖泊科学, $2003, \mathbf{1 5}(4): 331-338$. ]

[21] Lijklema L, Aalderink RH, Blom G et al. Sediment transport in shallow lakes, two case studies related to eutrophication. Transport and Transformation of Contaminants Near the Sediment-water Interface, 1994: 253-279.

[22] Thomas. JR, James M, Tim W et al. A sediment resuspension and water quality model of Lake Okeechobee. Journal of the American Water Resources Association, 1997, 33(3) : 661-678. DOI: 10.1111/j.1752-1688.1997.tb03540. x.

[23] Zhang Yunlin, Qin Boqiang, Chen Weimin et al. A study on total suspended matter in Lake Taihu. Resources \& Environment in the Yangtze Basin, 2004, 13(3): 266-271. [张运林, 秦伯强, 陈伟民等. 太湖水体中悬浮物研究. 长江流域 资源与环境, 2004, 13(3): 266-271.]

[24] Hu Chunhua, Hu Weiping, Zhang Fabing et al. Sediment resuspension in the Lake Taihu, China. Science Bulletin, 2006, 50(22): 2541-2545. [胡春华, 胡维平, 张发兵等. 太湖沉积物再悬浮观测. 科学通报, 2006, 50(22): 2541-2545.]

[25] Dou Guoren. Incipient motion of coarse and fine sediment. Journal of Sediment Research, 1999, (6) : 1-9. [窦国仁. 再论 泥沙起动流速. 泥沙研究, 1999, (6) : 1-9.] 